\title{
La narrativa de las políticas públicas en la jurisprudencia de la Corte Constitucional colombiana*
}

\author{
The narrative of public policy in the jurisprudence \\ of the Colombian Constitutional Court
}

\author{
Luisa Fernanda Cano Blandón ${ }^{* *}$
}

Recibido: 07/01/2014

Aprobado: 26/03/2014

Disponible en línea: 01/07/2014

\section{Resumen:}

Pese a que la referencia al Derecho en la teoría de las políticas públicas es escasa, en Colombia ha crecido el interés jurídico en este tema en los últimos años. En la vía inversa, los analistas de políticas públicas han prestado mayor atención al Derecho -y a los derechos- más allá de asumirlo como el marco jurídico institucional de toda política. Una de las razones de esta reciente conexión es la reiterativa referencia al concepto de políticas públicas en la jurisprudencia constitucional colombiana. El objetivo de este escrito es reconstruir la narrativa de la Corte Constitucional alrededor de la noción

\begin{abstract}
:
Although the reference to law on public policy theory is scarce, legal interest in this topic has increased in Colombia in recent years. One reason for this connection is the repetitive reference to the concept of public policy in constitutional jurisprudence. The aim of this paper is to reconstruct the narrative of the Colombian Constitutional Court about the notion of public policy since the promulgation of the 1991 Constitution until 2013. The approach to accomplish this task is the narrative analysis of public policy which is addressed primarily by Emery Roe (1994). The reconstruction of such narrative takes place in
\end{abstract}

doi:10.11144/Javeriana.PAPO19-2.nppj

\footnotetext{
* Artículo de investigación. El artículo es una actualización del marco teórico de la investigación "Eficacia de los derechos sociales: análisis de las sentencias de tutela y de las políticas públicas para su protección en el Magdalena Medio antioqueño" de la cual la autora fue investigadora principal. Este proyecto de investigación fue financiado por el Comité para el Desarrollo de la Investigación -CODI-y por la Dirección de Regionalización de la Universidad de Antioquia y el Acta de Finalización se firmó el 30 de septiembre del año 2010. Grupo de Investigación Gobierno y Asuntos Públicos, Universidad de Antioquia.

${ }^{* *}$ Abogada, Magíster en Gobierno y Asuntos Públicos de la Facultad Latinoamericana de Ciencias Sociales (FLACSO), sede México. Profesora de la Facultad de Derecho y Ciencias Políticas de la Universidad de Antioquia. Estudiante de Doctorado en Derecho de la Universidad de los Andes. Correo: If.cano10@uniandes.edu.co
} 
de políticas públicas desde la promulgación de la Constitución de 1991 hasta el año 2013. El referente para esta tarea es el análisis narrativo de políticas públicas sustentado principalmente por Emery Roe (1994).

La reconstrucción de tal narrativa se lleva a cabo en dos momentos: en primer lugar, se exploran los distintos escenarios en los cuales la Corte se refiere a las políticas públicas. En segundo lugar, se aborda el aspecto formal de su narrativa, es decir, los distintos modos cómo la Corte entiende las políticas públicas y cuál es la forma o acto jurídico a la que deben ser llevadas.

\section{Palabras clave:}

políticas públicas; Corte Constitucional; narrativa; escenarios fácticos; formalización

\section{Cómo citar este artículo:}

Cano, L. F. (2014). La narrativa de las políticas públicas en la jurisprudencia de la Corte Constitucional colombiana. Papel Político, 19(2). pp. 435-458. http://dx.doi. org/10.11144/Javeriana.PAPO19-2.nppj two stages: first, I explore different scenarios in which the Court refers to public policy in its narrative. Second, I analyze the formal aspect of the narrative, that is, the different ways how the Court understands the public policies and what is the legal form that these policies should take.

\section{Keywords:}

public policy; Constitutional Court; narrative; factual scenarios; formalization 


\section{Introducción}

Las políticas públicas pueden ser entendidas como una "serie de decisiones o acciones intencionalmente coherentes, tomadas por diferentes actores públicos y a veces no públicos, a fin de resolver de manera puntual un problema políticamente definido como colectivo" (Subirats y Knoepfel, 2008, p. 38). De tal definición es posible extraer su doble dimensión: como campo de estudio (policy field) y como instrumento de gobierno (policy-making process).

En la primera vía, las políticas públicas han sido consideradas en América Latina y particularmente en Colombia, como objeto de estudio de la ciencia política, la economía y la administración pública y solo en años recientes, el Derecho ha ingresado a formar parte de este campo multidisciplinar. Situación similar ha ocurrido en la segunda dimensión, toda vez que los policy makers, empleados en agencias del Estado nacional o local, tradicionalmente han sido personas con conocimientos técnicos de las disciplinas mencionadas y difícilmente, ha sido un campo laboral propio de los abogados ${ }^{1}$.

Sin embargo, casi de forma paralela al desarrollo del campo de las políticas públicas, Dahl (1957) advirtió que es ingenuo considerar a los tribunales constitucionales como instituciones estrictamente jurídicas y que, precisamente, una de sus funciones es trazar políticas públicas.

A propósito de lo señalado por Dahl (1957), en Colombia el interés jurídico por las dos dimensiones de las políticas públicas -es decir, por su estudio y su diseño- ha crecido en gran medida, debido a que la Corte Constitucional comenzó a usar con frecuencia este concepto en sus sentencias y a llenar de contenido una expresión que, en esencia, no pertenece a ninguna categoría jurídica particular, lo cual no ha sido impedimento para que la Corte se involucre, de diversas maneras, en el proceso de políticas públicas.

El objetivo de este escrito es reconstruir la narrativa de la Corte Constitucional colombiana alrededor de la noción de políticas públicas desde la promulgación de la Constitución de 1991. La reconstrucción de tal narrativa se realiza a partir de dos categorías fundamentales: a) Los distintos escenarios fácticos, es decir, los hechos y circunstancias en los cuales la jurisprudencia constitucional alude a las políticas públicas y b) La forma en que se refiere a tal concepto.

\footnotetext{
${ }^{1}$ Lo anterior ha sucedido por varias razones. De un lado los teóricos de las políticas públicas pocas veces han mencionado la dimensión jurídica de las políticas y tampoco se han detenido en la relación entre políticas públicas y derechos. En tal perspectiva, el derecho aparece como el marco regulador en el que se produce un juego de actores racionales que utilizan la norma como una variable más dentro del complejo proceso de las políticas, es decir, como el marco institucional en el que se producen los intercambios y que permite claridad en los derechos de propiedad y reducción de costos de transacción. De otro lado, la doctrina jurídica ha ignorado por largo tiempo su posición frente a las políticas públicas por considerar que pertenecen a otros campos de estudio por lo que, de modo general, hasta hace pocos años este tema no se había considerado objeto de estudio jurídico .
} 
No se trata, por tanto, de ahondar en el debate sobre los efectos y formas del activismo judicial en distintas políticas públicas o a la manera en que la Corte se ha comportado como un policy maker ${ }^{2}$. Se trata más bien, de un intento por ponerle piso a tal discusión, debido a que la jurisprudencia constitucional ha utilizado la expresión 'políticas públicas' de diversas maneras en numerosas decisiones, las cuales fueron revisadas en el desarrollo de esta investigación.

El estudio se justifica en la medida en que el concepto de política pública no es un término prescriptivo del que se conozca un único significado y un solo uso posible; por el contrario, su comprensión ha dependido en parte, del lente disciplinar que se utilice. Explorar el significado jurisprudencial de las políticas públicas nos permitirá entonces tener idea de cuándo y cómo la Corte Constitucional ha echado mano de este concepto.

Las hipótesis que orientaron la investigación son:

1. El principal escenario fáctico que subyace al uso de las políticas públicas en la jurisprudencia de la Corte Constitucional es el de la violación a la faceta prestacional y progresiva de los derechos constitucionales.

2. El uso jurisprudencial de las políticas públicas obliga principalmente al gobierno como institución competente para su diseño.

3. Las políticas públicas son asimiladas en la jurisprudencia de la Corte a conceptos cercanos como políticas de Estado, planes y programas.

Desde el punto de vista metodológico la investigación se apoya en la perspectiva del análisis narrativo de políticas públicas (Narrative policy analysis) propuesto por Emery Roe (1994), que se inscribe dentro del llamado giro argumentativo de las políticas públicas y estima que alrededor de cada política se tejen relatos (stories) elaborados por distintos actores. En tal sentido, esta investigación considera que la Corte Constitucional es un actor más en el campo de las políticas públicas, por lo cual construye su propio relato que puede entrar en pugna con otros actores. En esta ocasión, el propósito es develar la narrativa alrededor del concepto mismo que tiene la Corte sobre lo que es una política pública.

El estudio se desarrolló con base en el contenido de decisiones de la Corte Constitucional $^{3}$ para lo cual se confrontaron distintas fuentes ${ }^{4}$. La búsqueda arrojó un total de 94 decisiones repartidas así: 63 sentencias de tutela (T), dos sentencias de unificación

\footnotetext{
${ }^{2}$ Al respecto ver, entre otros: Abramovich y Pautassi (2009), Garavito, Arcidiácono y Espejo (2010), Cano Blandón (2012) y Sierra Cadena (2009).

${ }^{3}$ La búsqueda se realizó igualmente en el Consejo de Estado y en la Corte Suprema de Justicia, pero los hallazgos fueron muy precarios. En la Corte Suprema de Justicia se encontraron cuatro sentencias de tutela, todas de la Sala Laboral y que se refieren a las políticas públicas como ámbito propio de decisiones políticas y no judiciales. En el Consejo de Estado se encontraron ocho sentencias referidas de algún modo a la materia.

${ }^{4}$ En primer lugar se consultó en la Relatoría de la Corte Constitucional. La información encontrada se comparó con el buscador Lex Base y con el buscador de jurisprudencia del Centro de
} 
(SU), 18 de constitucionalidad (C) y once autos (A). La información fundamental de cada decisión se documentó en una base de datos. El objetivo sin embargo, no es mostrar las tendencias en el uso del término de manera diacrónica. En su lugar, se presenta la narrativa a partir del contexto o escenario fáctico en que la Corte se refiere a las políticas públicas y a partir de las formas o instrumentos donde la Corte estima que se plasma -o se debe plasmar- su contenido.

El artículo consta de tres partes. En la primera se profundiza en la base metodológica que orientó la investigación a partir del análisis narrativo de las políticas públicas. En la segunda parte se exploran los escenarios fácticos en los cuales la Corte Constitucional se refiere a las políticas públicas y finalmente, se alude a su aspecto formal, es decir, a las distintas maneras en que se entienden las políticas públicas en las decisiones examinadas.

\section{El análisis narrativo de las políticas públicas}

El análisis de políticas públicas es la actividad que permite estudiar e interpretar el proceso de construcción y ejecución de una determinada política pública. Como indica Roth (2010) “tal como para cualquier objeto de estudio, para analizar las políticas públicas es necesario disponer de un marco teórico y conceptual y de herramientas que nos sirvan como lente o tamiz para orientar la labor investigativa" (p. 17). Son estos lentes o enfoques los que hacen posible elaborar una explicación de este objeto de estudio y tener un mejor conocimiento de los elementos que inciden en el proceso de una política pública.

Los mencionados enfoques de análisis de las políticas públicas corresponden a distintos paradigmas propios de las Ciencias Sociales (Roth, 2007). Así, los modelos de elección racional, secuencial y neoinstitucional pueden inscribirse dentro de un paradigma pospositivista -e incluso positivista- mientras que el enfoque cognitivo correspondería a una postura epistemológica constructivista. Además de los modelos cognitivos, el paradigma constructivista también sustenta lo que en años recientes se ha denominado el "giro argumentativo" (Fisher, 1996) dentro del cual es posible adscribir enfoques como el discursivo (Fischer, 1996), el retórico (Majone, 2005) y el que interesa para efectos de esta investigación, el enfoque narrativo del Policy Analysis (Roe, 1994).

Un punto común entre los modelos inspirados en el giro argumentativo es considerar que "la política pública está hecha de palabras" (Majone, 2005, p. 35) que deben ser tenidas en cuenta por el analista. Según Gottweis (2007) el término argumentative policy analysis subsume un grupo diferente de enfoques que rescatan el lenguaje como una característica determinante del análisis de políticas públicas y comparten la especial

Documentación Socio-Jurídica de la Rama Judicial (CENDOJ) http://200.74.129.85/Jurisprudencia/ consulta/index.html 
atención sobre la argumentación y el uso, movilización y significación de los argumentos y los símbolos tanto en el policy making como en el policy analysis.

En efecto, el giro argumentativo privilegia la racionalidad argumentativa o discursiva sobre la racionalidad instrumental; ofrece un modelo de comunicación dialéctica de la toma de decisiones políticas y la sustenta en una lógica informal de la razón práctica que se erige en contra de la lógica formal del neopositivismo. Intenta superar por tanto, la limitación epistemológica del empiricismo en el análisis de políticas y el proceso de decisión tecnocrático y lineal que de él se deriva. De este modo, el análisis argumentativo se centra en el papel crucial del lenguaje, la argumentación, la retórica y las historias en la elaboración del debate, así como en la estructuración del marco deliberativo en el que se formula la política.

Como se mencionó, dentro de este argumentative policy analysis se inscribe el enfoque narrativo, el cual estima que las políticas son resultado del encuentro de historias, narrativas y discursos que dan significado a la realidad. El enfoque narrativo sugiere que alrededor de cada política pública se tejen relatos (stories), generalmente en pugna, que evidencian la polarización propia de los asuntos públicos. Esto no solo explica la dinámica del proceso de la política pública, al demostrar la presencia de narrativas múltiples y contradictorias, sino que además, otorga igual peso a las narrativas de los actores, de forma que distintas versiones de un mismo asunto sean consideradas en el proceso de formulación de las políticas, particularmente en los asuntos complejos e inciertos y que suscitan polarización.

El análisis de las narrativas se refiere a una rama de la teoría literaria conocida como la 'narratología' que se ocupa de estudiar relatos, textos (hablados o escritos) y narraciones, es decir, secuencias de eventos que dan cuenta de la transición de un estado a otro (Van Eeten, 2007). Así, a partir del estudio del texto, el análisis narrativo trata de dar sentido a la forma en que un relato es comunicado.

Si bien esta investigación no se ocupa de analizar el proceso de una política pública en particular, el enfoque narrativo resulta pertinente debido a que la Corte Constitucional se ha comportado, en numerosas ocasiones, como un actor de políticas públicas, asunto que amerita la reconstrucción del concepto en sus decisiones. Las providencias analizadas por tanto, son asumidas como relatos que se hilan en una narrativa alrededor de las dos categorías ya mencionadas: los escenarios fácticos y la formalización de las políticas públicas. La intención de los siguientes puntos es dar sentido a los relatos a partir de estos dos elementos.

\section{Los escenarios fácticos de las políticas públicas en la jurisprudencia}

Se identificaron dos escenarios fundamentales en los que la Corte Constitucional alude a las políticas públicas: $i$ ). Cuando reconoce la necesidad de formular políticas que desarrollen la faceta prestacional de los derechos y ii). Cuando declara un estado de cosas inconstitucionales (ECI). Ambos escenarios no son excluyentes, es decir que es posible 
encontrar ambos argumentos en una misma providencia, sin embargo estos contextos se separan en el escrito para resaltar que no siempre que la Corte adopta órdenes complejas $^{5}$ y fallos estructurales -que involucran políticas públicas- se ha declarado un ECI.

\section{La faceta prestacional de los derechos constitucionales}

La Corte Constitucional ha insistido en que todo derecho fundamental exhibe dos facetas. La primera es una faceta de abstención y se refiere a la protección del contenido del derecho impidiendo que terceros lo transgredan. La segunda es una faceta de acción que ordena contar con mecanismos idóneos para garantizar su goce efectivo (Sentencia T-133/o6).

En este escenario la Corte entiende que el diseño de políticas públicas está dirigido al segundo propósito, es decir a la satisfacción de la faceta asistencial, prestacional o programática de los derechos. Se trata, por tanto, de una obligación estatal derivada del modelo de Estado Social ${ }^{6}$. Este es el contexto más recurrente en el que la Corte se refiere a las políticas públicas y el primero al que alude en el año 1992.

Así, en sentencia T-533-92 se indicó que "De manera general, puede decirse que una ley, expedida por el Congreso de la República, preside el desarrollo de la política pública mediante la cual se busca la efectividad de un determinado derecho asistencial" (T-533-1992). En similar sentido, resalta el salvamento de voto del magistrado Eduardo Cifuentes en 1999 cuando advirtió que la eficacia de los Derechos Económicos, Sociales y Culturales depende de la definición de políticas públicas que los desarrollen, puesto que carecen de aplicación inmediata (T-177-99).

Pero sin duda la sentencia que relacionó explícitamente las políticas públicas con la protección de los derechos por primera vez fue la T-595-02, en la cual la Corte resolvió el caso del acceso de las personas en situación de discapacidad al sistema integrado de transporte público Transmilenio. En esa ocasión la Corte sostuvo que "la integración social de personas como el accionante constituye un problema público que ha de ser atendido mediante una política pública” (T-595-02).

Esta sentencia caracteriza, además, por disponer tres elementos mínimos que debe tener toda política pública y que son reiterados en la jurisprudencia desde entonces:

\footnotetext{
${ }^{5}$ Según la Corte Constitucional con 'órdenes complejas' se refiere a "la fórmula utilizada en casos en los que se trata de amparar la dimensión progresiva de un derecho fundamental, por lo que resulta necesaria la concurrencia de distintas autoridades en la solución del conflicto" (T-1259-08). ${ }^{6}$ En la sentencia T-630-08 se dijo al respecto que "la realización de la fórmula del Estado social de derecho [...] comporta el deber de adoptar y realizar políticas públicas encaminadas a promover condiciones de vida dignas para todos, en especial para aquellos que no alcanzan a satisfacer los mínimos exigibles, solventando de esta manera la marginalidad, la exclusión y la desigualdad a que se hallan expuestos."
} 
[...] (i) que la política efectivamente exista; (ii) que la finalidad de la política pública debe tener como prioridad garantizar el goce efectivo del derecho; y (iii) que los procesos de decisión, elaboración, implementación y evaluación de la política pública permitan la participación democrática. (T-595-02)

En este primer escenario de la narrativa jurisprudencial sobre políticas públicas, abundan las decisiones judiciales que protegen la faceta programática de los derechos de poblaciones vulnerables?: discapacitados ${ }^{8}$, niños y niñas ${ }^{9}$, población desplazada ${ }^{10}$, minorías étnicas ${ }^{11}$, población LGBTI ${ }^{12}$, vendedores ambulantes ${ }^{13}$ y recicladores ${ }^{14}$, entre otros. Frente a estos grupos poblacionales, la Corte advierte dos tipos de obligaciones estatales derivadas de la cláusula de erradicación de las injusticias presentes: un deber positivo de implementar políticas encaminadas a lograr la igualdad real de la población y un deber negativo que le ordena abstenerse de adoptar políticas regresivas que impliquen un retroceso para los derechos de los afectados por una decisión pública ${ }^{15}$.

La Corte, de otro lado, deriva la obligación de diseñar políticas públicas del texto mismo de la Constitución:

La Constitución ordena que en la formulación de políticas públicas, así como en su implementación y evaluación, se incorpore específicamente el goce efectivo de los derechos y principios constitucionales, lo cual no impide que las autoridades competentes democráticamente elegidas fijen prioridades y definan, dentro de los márgenes de configuración que les son propios, el contenido de tales políticas públicas (C-423-05).

\footnotetext{
${ }^{7}$ En tal sentido la sentencia T-585-08 indica que "Los sujetos en condición de debilidad manifiesta son los principales destinatarios de políticas públicas que aseguren el goce efectivo de los derechos económicos, sociales y culturales".

${ }^{8} \mathrm{Al}$ respecto las sentencias T-638-99, T-823-99, T-595-02, T-149-02, T-617-05, T-061-06, T-124808, T-777-09, T-765-11, T-285-12.

${ }^{9} \mathrm{Al}$ respecto las sentencias T-587-98, T-220-04, T-826-04, T-1030-06, T-170-07, T-1259-08, SU225-98, T-988-08, T-321-08.

${ }^{10} \mathrm{Al}$ respecto las sentencias T-602-03, T-025-04, T-919-06, T-966-07, T-821-07, T-725-08, T- 56008, T-817-08, T-038-09, T-479-11, T-675-11, y los Autos 027-07, 005, 007, 008 de 2009, 382, 383, 385 de 2010.

${ }^{11} \mathrm{Al}$ respecto las sentencias C-175-09, T-113-09, T-514-09.

${ }^{12} \mathrm{Al}$ respecto la sentencia T-314-11.

${ }^{13} \mathrm{Al}$ respecto las sentencias T-772-03, T-465-06, T-729-06, T-773-07, T-630-08, T-1179-08, T-77509, T-244-12.

${ }^{14} \mathrm{Al}$ respecto las sentencias T-411-09, T-291-09.

${ }^{15}$ Este grupo está conformado por las sentencias T-772-03, T-826-04, T-025-04, C-174-04, C-42305, T-411- 09, T-291-09, T-479-11.
} 
Por otra parte, la sentencia C-646-01 resulta de especial relevancia para la reconstrucción de la narrativa jurisprudencial sobre políticas públicas, pues contiene una amplia referencia teórica al tema. En esta providencia la Corte se da a la tarea de explorar cada uno de los artículos de la Constitución que, de algún modo, se refieren a las políticas públicas y encuentra quince expresiones que aluden al sentido de policy (y no politics) del término 'política':

\begin{abstract}
Dentro del concepto de diseño de una política pública se encuentra la acción de articularla jurídicamente, es decir, de plasmar los elementos de la política en un conjunto de proyectos normativos. Como la Constitución emplea frecuentemente el concepto de "política" para designar, no la actividad electoral sino las medidas atinentes a las "políticas públicas", resulta pertinente analizar las implicaciones jurídicas de la incorporación de este concepto a la Constitución (C-646-01) ${ }^{16}$.
\end{abstract}

En este punto vale la pena preguntarse si, en efecto, el Constituyente de 1991 contempló, de alguna manera, la relación entre derechos constitucionales y políticas públicas. Pese a que se consultaron distintas fuentes de la época no se halló ningún resultado positivo, por lo que todo indica que esta relación derivó más bien de la interpretación de la Corte que de la voluntad del Constituyente primario.

La Corte, así mismo, extrae la obligación de desarrollar políticas públicas, respecto a sujetos tradicionalmente marginados o discriminados, del inciso segundo del artículo

\footnotetext{
16 "Ante todo, subraya la Corte que el concepto de políticas públicas fue constitucionalizado en varias disposiciones de la Carta y en relación con diversos ámbitos de la actividad estatal. Por ejemplo, la Constitución se refiere a: (a) "la política exterior de Colombia [que] se orientará hacia la integración latinoamericana y del Caribe" (art. 9); (b) a las "políticas para la prestación de servicios de salud por entidades privadas" (art. 29 inciso 2 y art. 49); (c) a "una política de previsión, rehabilitación e integración social para los disminuidos físicos, sensoriales y psíquicos, a quienes se prestará la atención especializada que requieran" (art. 47); (d) a las "políticas salariales y laborales" (art. 56, inciso 2); (e) a la "política ... en materia de televisión" (art. 77); (f) a la "política comercial" (art. 150 \# 19 literal c); (g) a las "las políticas atinentes al despacho (de los Ministros)," (art. 208); (h) a las "políticas para [...] [la] enseñanza de los derechos humanos" (art. 282 \# 2); (i) a "las políticas y los planes y programas de desarrollo económico y social dentro (del) territorio [indígena], (art. 330 \# 2); (j) a que "los desacuerdos con el contenido de la parte general [del Plan Nacional de Desarrollo], si los hubiere, no serán obstáculo para que el gobierno ejecute las políticas propuestas en lo que sea de su competencia" (art. 341 inciso 2); (k) a los "sistemas de evaluación de gestión y resultados de la administración pública, tanto en lo relacionado con políticas como con proyectos de inversión" (art. 343); (I) a las "políticas generales de administración y control de eficiencia de los servicios públicos domiciliarios" (art. 370 y art. 48 transitorio); (m) a la "política económica general" (art. 371 inc. 2); (n) a las "políticas a... cargo" del Banco de la República (art. 371 inc. 3); (ñ) a las "políticas económica, social y ambiental que serán adoptadas por el gobierno" (art. 339); (o) a la "política de reconciliación" (art. 30 transitorio) y (p) a la "política del Estado en materia criminal" (art. 251, \#3) (Sentencia C-646-01).
} 
13 constitucional que afirma que el Estado debe promover las condiciones para que la igualdad sea real y efectiva y adoptar medidas en favor de grupos discriminados o marginados. En palabras de la Corte:

\section{[...] el constituyente ha ordenado el diseño de una política pública orientada a lograr su rehabili- tación, integración social y procurarles la atención especializada que sus necesidades demanden; política pública que el legislador desarrolló con la expedición de la Ley 361 de 1997 (T-449-08).}

Mención aparte merece la alusión de la Corte a las políticas públicas como sinónimo de acciones afirmativas a favor de grupos tradicionalmente discriminados ${ }^{17} \mathrm{y}$ como escenario de consulta previa para los pueblos indígenas ${ }^{18}$. En el primer caso vale la pena mencionar una providencia reciente en la que protege el derecho al trabajo y al mínimo vital de una mujer que no había sido tenido en cuenta en un proceso de reubicación. Al respecto la Sala señaló que:

[...] una política pública dirigida a la formalización de la economía como apoyo a las personas que ocupan el espacio público objeto de recuperación, debe tener una perspectiva con enfoque diferencial, de tal forma que en los censos se oiga también la voz de las mujeres (T-386-13).

Valga mencionar, por último, que en principio la Corte se refiere a la relación entre políticas públicas y un cierto tipo de derechos -los Derechos Económicos, Sociales y Culturales ${ }^{19}$ - pero en los últimos años ha advertido que se trata de un mecanismo para atender la faceta prestacional de cualquier derecho constitucional y que son expresión del mandato de progresividad de todos los derechos ${ }^{20}$.

17 "Como lo ha explicado la Corte en numerosas ocasiones con la expresión acciones afirmativas o de diferenciación positiva se designan políticas o medidas dirigidas a favorecer a determinadas personas o grupos, ya sea con el fin de eliminar o reducir las desigualdades" (C-174-04). 18 "Los pueblos indígenas y tribales deben contar con mecanismos que les permitan la participación en todos los niveles de adopción de políticas estatales que les conciernan" (C-175-09). En similar sentido la T-376-12 dice que "el proceso de consulta está destinado a establecer el alcance de la concesión, entendida como un proyecto o medida que hace parte de una política pública adelantada en el Distrito Turístico de Cartagena, asegurando en todo momento la participación de todos los interesados".

${ }^{19}$ Así lo indica en sentencia T-675-11: "En el estado social de derecho, el compromiso con la garantía efectiva de los derechos económicos, sociales y culturales consagrados en la Carta, tales como el derecho a la salud, a la seguridad social, a la educación, al acceso al agua potable, al trabajo, o a la vivienda digna implica, para su efectivo cumplimiento, que el Estado implemente políticas públicas, encaminadas a la obtención de los recursos necesarios para su materialización." ${ }^{20}$ En ocasiones la Corte ha entendido que la progresividad se predica de las políticas, aunque es una característica propia de los derechos que se puede cumplir a partir de las políticas. En la sentencia C- 443-09 expresó "Solo será objeto de análisis el cargo relacionado con la vulneración del principio de progresividad de las políticas públicas en materia de protección al derecho al medio ambiente sano". 


\section{Las políticas públicas como consecuencia de la declaratoria del Estado de Cosas Inconstitucional}

Cuando la Corte ha declarado la violación sistemática, masiva y generalizada de derechos constitucionales, es decir, un estado de cosas inconstitucional (ECI), la parte resolutiva de la providencia se ha acompañado generalmente, de órdenes que implican la adopción de medidas de política pública aunque explícitamente no se aluda al término. Esto significa que la declaratoria de un ECI es un fallo estructural pero existen fallos estructurales que no conllevan tal declaratoria. Incluso la Corte ha sido más enfática en sus órdenes de política pública en decisiones en las que no ha declarado un ECI, tal como ocurrió con la sentencia T-760 de 2008 relativa al derecho a la salud.

En 1997 la Corte declaró por primera vez un estado de cosas contrario a la Constitución debido a que varios docentes del departamento de Bolívar no fueron afiliados a ningún Fondo de Prestación Social a pesar de que el empleador hacía el descuento pertinente a su salario (SU-559-97). El año siguiente se declaró tal estado en varias circunstancias: frente al problema carcelario en el país (T-153-98 ${ }^{21}$, a raíz de la situación de amenaza de los defensores de derechos humanos (T-590-98 $)^{22}$, por la no convocatoria a concurso para la designación de notarios en propiedad (SU-250-98) ${ }^{23}$, por el no pago de salarios a docentes (T-289-98), por el no pago de las mesadas pensionales (T-559-98) ${ }^{24}$ y por la demora en responder las solicitudes de pensión de jubilación de la Caja Nacional de Previsión Social (Cajanal) (T-068-98, T-1234-08).

Estas primeras sentencias que declaran un ECI contienen órdenes tímidas a diferencia de las que se comienzan a adoptar a raíz de la precisión doctrinal que hace la sentencia T-025 de 2004 sobre el alcance del ECI ${ }^{25}$. En todo caso como ya se indicó, no ha sido necesario declarar tal situación para que la parte resolutiva contenga órdenes complejas que involucren a distintas autoridades.

\footnotetext{
${ }^{21}$ Sobre el mismo tema ver sentencias T-1291-00, T-1030-03, T-1096-04.

22 Reiteran tal estado de cosas las sentencias T-558-03 y T-1191-04.

${ }^{23}$ Sobre esta misma declaratoria ver sentencias 1695-00, C-373-02, C-076-06, C-421-06.

${ }^{24}$ Posteriormente sobre el mismo tema las sentencias T-525-1999 y T-547-01.

${ }^{25}$ En esta sentencia se indicaron los factores que se deben tener en cuenta para hablar de un ECI: "1) La vulneración masiva y generalizada de varios derechos constitucionales que afecta a un número significativo de personas, 2) La prolongada omisión de las autoridades en el cumplimiento de sus obligaciones para garantizar los derechos, 3) La adopción de prácticas inconstitucionales, como la incorporación de la acción de tutela como parte del procedimiento para garantizar el derecho conculcado, 4) La no expedición de medidas legislativas, administrativas o presupuestales necesarias para evitar la vulneración de los derechos, 5) La existencia de un problema social cuya solución compromete la intervención de varias entidades, requiere la adopción de un conjunto complejo y coordinado de acciones y exige un nivel de recursos que demanda un esfuerzo presupuestal adicional importante, 6) EI hecho de que si todas las personas afectadas por el mismo problema acudieran a la acción de tutela para obtener la protección de sus derechos, se produciría una mayor congestión judicial" (T-025-04).
} 
Para concluir este apartado, vale la pena mencionar dos aclaraciones importantes que hace la Corte en el tema. La primera es que si bien la garantía de la faceta prestacional de todos los derechos requiere la ejecución de políticas públicas, no todas las políticas están directamente relacionadas con la satisfacción de derechos ni en todas las políticas es conveniente la intervención de la Corte Constitucional. Así lo precisa el Auto 385-10:

\footnotetext{
[...] el juez constitucional no se encuentra facultado para intervenir per se y de manera ilimitada en las políticas públicas del gobierno, sino únicamente en la medida en que tengan una relación de conexidad directa y estrecha con la vigencia de los derechos constitucionales de la población afectada.
}

De esta forma la Corte parece limitar su competencia en el campo de las políticas públicas únicamente a los casos relacionados directamente con derechos constitucionales. Sin embargo, es difícil pensar en políticas públicas ajenas a la garantía de los derechos, máxime cuando su razón de ser es, precisamente, avanzar en el cumplimiento de las obligaciones del Estado.

La segunda aclaración que hace la Corte es que el hecho de tener una política pública no garantiza el cumplimiento de los derechos, por lo que indica que:

[...] la mera demostración de ajustes presupuestales, la expedición de normas o el diseño
y ejecución parcial de políticas públicas, no constituye, en modo alguno, un motivo o una
razón concluyente para dar por superado el estado de cosas inconstitucional (Auto 385-10).

Si bien no contar con una política pública que atienda la faceta prestacional de los derechos es considerado por la Corte como un indicio de inconstitucionalidad, el hecho de que esta exista o se encuentre plasmada en algún documento no resuelve los problemas mágicamente. La política pública hace parte de un proceso que, en no pocas ocasiones, se inicia con el diseño de sus programas y proyectos pero cuyo éxito depende, principalmente, de la etapa de implementación; de lo contrario la política, al igual que ocurre con muchas leyes, no serán más que letra muerta. Es por eso que la Corte Constitucional se ha ocupado de la formalización de las políticas de tal manera que vinculen, con mayor claridad y de manera perentoria, a sus responsables.

\section{La formalización de las políticas públicas de acuerdo a la narrativa jurisprudencial}

Tal como se mencionó al inicio, la reconstrucción de la narrativa jurisprudencial en este caso, se interesa por los escenarios fácticos en que aparecen las políticas públicas pero también por el aspecto formal, es decir, interesa conocer, de acuerdo con la interpretación 
de la Corte, el grado de formalización -si se quiere de juridificación- que tienen las políticas públicas en tal narrativa.

En la primera parte se explora la narrativa jurisprudencial que estima que las políticas públicas están vinculadas directamente con la función administrativa de la rama ejecutiva; en la segunda se presenta su grado de vinculación con el legislativo.

\section{Las políticas como actos administrativos}

En la mayoría de decisiones analizadas, las políticas públicas son consideradas un deber del ejecutivo en desarrollo de la ley. En su jurisprudencia, la Corte alude indistintamente a política pública como "potestad reglamentaria”,26, "política legislativa o reglamentaria”"27, "política estatal”28, "política institucional” ${ }^{29}$, planes ${ }^{30}, \operatorname{programas}^{31}$ y proyectos ${ }^{32}$.

Asimismo, la Corte aborda la relación entre términos muy cercanos de forma diversa, por lo que valdría la pena preguntarse: ¿Para la Corte los planes de desarrollo contienen políticas públicas? Y, des lo mismo una política pública que un programa? Si bien no existe una única manera de abordar esta relación, la doctrina ha sugerido que

${ }^{26}$ Esto queda claro en la sentencia T-602-03: "es indispensable que la Corte analice las políticas públicas realmente existentes en materia de desplazamiento forzado, pues no puede obviarse que el desarrollo de los derechos de contenido prestacional es básicamente progresivo y, en principio, está atribuido al legislador, así como, por vía reglamentaria, al ejecutivo".

${ }^{27} \mathrm{Al}$ respecto la sentencia T-533-92: "La pobreza, sin duda, atenta contra la vigencia efectiva de los derechos fundamentales. Sus causas estructurales son combatidas mediante políticas legislativas". Así mismo la T-777-09 indica frente a la seguridad social "la necesaria adopción de políticas legislativas o reglamentarias o técnicas que determinen de manera precisa las prestaciones exigibles y las condiciones para acceder a las mismas; las instituciones obligadas a brindarlas y su forma de financiación". ${ }^{28}$ Al respecto la sentencia T-499-95 señala: "El subsidio de vivienda, materia de esta controversia, se relaciona con la política estatal de apoyo y protección a las personas y sectores más vulnerables de la población colombiana". En similar sentido la T-025-04 y la T-1037-10.

${ }^{29} \mathrm{Al}$ respecto la T-638-99: "resulta pertinente hacer algunas precisiones atinentes al tema de la educación como derecho fundamental; a la educación especial para personas con limitaciones auditivas y, finalmente, a la ejecución de las políticas institucionales sobre la materia."

${ }^{30} \mathrm{Al}$ respecto la sentencia T- 595-02: "El carácter progresivo de la prestación no puede ser invocado para justificar la inacción continuada, ni mucho menos absoluta, del Estado. Primero, debe existir una política pública, generalmente plasmada en un plan." En similar sentido la T- 1259-08: "para esta Sala la ausencia total de un plan estatal dirigido a dar solución a la problemática a la que se ha hecho referencia en esta providencia, constituye una clara violación de los derechos fundamentales de los menores".

${ }^{31}$ Al respecto la sentencia T-149-02 "La ambigüedad o la incoherencia de los fines del programa, la incertidumbre suscitada por una evidencia empírica insuficiente o la no sostenibilidad económica del programa amenazan con frustrar la debida prestación de la seguridad social". En sentido similar el Auto 382-10 ordena "diseñar e implementar de manera urgente un programa de intervención y atención en salud, nutrición y seguridad alimentaria para atender al pueblo Hitnu o Macaguán". ${ }^{32}$ Al respecto la sentencia T-772-03 indica: "La anterior política se ha materializado en una serie de programas y proyectos; entre ellos, los de reubicación para los vendedores informales". 
en los planes de desarrollo "no encontramos propiamente políticas públicas [sino] lineamientos generales para estas” (Cuervo, 2007, p.76). Las políticas contienen programas, estos a su vez proyectos y la unidad mínima de análisis está constituida por acciones.

En la sentencia C-507-08 parece que la Corte adopta la relación sugerida en la Gráfica 1 al indicar que toda política pública del sector central debe encontrarse reflejada en el plan de desarrollo ${ }^{33}$, sin embargo en otras ocasiones sostiene que las políticas públicas 'obedecen' a planes y programas ${ }^{34}$, en otras se entiende que las políticas se implementan mediante planes y programas ${ }^{35}$, que la potestad reglamentaria desarrolla las políticas públicas $^{36}$, o que estas se reglamentan mediante actos administrativos ${ }^{37}$ de manera que su narrativa en el tema no ha sido uniforme.

De otro lado, en materia de protección a la población desplazada parece sugerir la necesidad de que exista una política pública por cada derecho constitucional y no una sola política con distintos programas para atenderlos ${ }^{38}$.

\section{Gráfica 1}

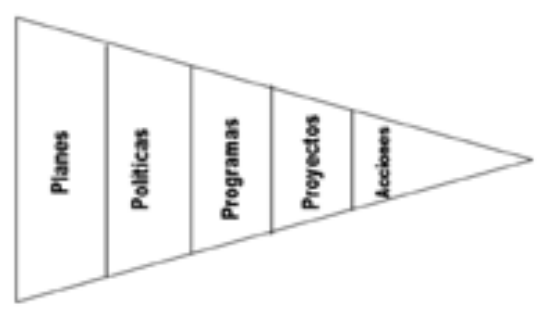

Fuente: elaboración propia con base en Roth, André Nöel (2006)

\footnotetext{
${ }^{33} \mathrm{Al}$ respecto la sentencia C-507-08 y lo mismo ocurre en la sentencia T-765-11 en la que se advierte que "la niñez debe crecer con apoyo en políticas públicas, basadas en programas de prevención y protección".

${ }^{34} \mathrm{Al}$ respecto la sentencia T-617-2005: "No desconoce esta Sala la necesidad de que las políticas públicas de vivienda obedezcan a planes y programas que señalen requisitos los que se tendrán que cumplir". ${ }^{35}$ Al respecto la sentencia T-966-2007 en la que se ordena convocar "a una mesa de trabajo específica para discutir los problemas de vivienda de las personas desplazadas asentadas en esa población, en donde se revisen las políticas de atención en la materia de cada entidad, así como los planes y programas previstos para su implementación".

${ }^{36} \mathrm{Al}$ respecto la sentencia T-760-08: "Es pues imprescindible para garantizar el goce efectivo del derecho a la salud de las personas, que el Estado cumpla con su obligación de crear la reglamentación adecuada para posibilitar la implementación de las políticas públicas en salud".

${ }^{37}$ Al respecto la sentencia T-499-95: "la entidad o entidades directamente responsables del manejo de una determinada política pública, pueden expedir actos administrativos que regulan los requisitos, trámites y procedimientos a los que deben someterse los eventuales beneficiarios de las prestaciones que hayan de distribuirse".

${ }^{38}$ El Auto 008 de 2009 le exige al gobierno la "Demostración de que las políticas públicas relacionadas con cada uno de los derechos constitucionales de los desplazados conducen efectivamente a lograr el goce efectivo de los derechos por parte de los desplazados".
} 
Lo cierto es que a la Corte no le basta con hablar de políticas públicas, cuando lo hace, generalmente acompaña este concepto de expresiones adicionales para reforzar su intención de dirigir a las entidades competentes a la acción pública ${ }^{39}$.

En cuanto a la formalización de la política, hay una aparente contradicción en la narrativa sobre el tema. Por un lado, la Corte indica que no se trata solamente de un texto escrito: "Una política pública no es el conjunto de promesas y de directrices que una autoridad pública formula en un texto. Una política pública la constituye el conjunto de acciones y omisiones de una autoridad sobre una cuestión" (T-113-09) ${ }^{40}$. En contraste, en el Auto 007-09 relativo a la población desplazada, ordena que la política pública se formalice mediante un acto administrativo ${ }^{41} \mathrm{y}$ en otro caso relativo al derecho a la educación, exige que la política sea escrita con el fin de verificar el cumplimiento de su orden ${ }^{42}$.

Esta contradicción sin embargo, es solo aparente porque la Corte se refiere a dos asuntos distintos. Si bien es importante que los compromisos, plazos y responsables de una política determinada queden escritos en algún documento o acto administrativo, esto solo constituye la etapa de diseño de la política pública pero la Corte insta a culminar la etapa de ejecución, es decir, a proceder con el conjunto de acciones que se deben implementar para hacer frente a una problemática.

El grado de formalización exigido en las providencias mencionadas se relaja en la sentencia C-646-01, donde precisó que la política no necesariamente tiene que estar plasmada en un instrumento jurídico ya que puede tratarse de un documento político. Cuando se opta por el primer caso, la política puede estar contenida en un acto

\footnotetext{
39 "Entre otros deberes que surgen para el Estado a partir de dicha declaración se encuentran, por una parte, aquellos relativos a la adopción e implementación de políticas, programas y medidas positivas encaminadas al logro de una igualdad real de condiciones y oportunidades entre los asociados" Sentencia T-038-09. En similar sentido la sentencia T-045-2010 apunta: "Este Tribunal ordena al Ministerio de la Protección Social [...] diseñe e implemente los protocolos, programas y políticas necesarias de atención en salud que respondan a las necesidades particulares de las víctimas del conflicto armado y sus familias".

${ }^{40}$ En esa sentencia la Corte precisó: "la política del Ejército Nacional en materia de reclutamiento de poblaciones indígenas no la constituye un texto o un documento, sin importar cuál sea este, sino lo que en la realidad hace y deja de hacer la institución castrense a través de sus distintos miembros, en materia de reclutamiento de jóvenes indígenas". (T-113-09)

${ }^{41}$ Dicho Auto ordena "al Consejo Nacional para la Atención Integral a la Población Desplazada por la Violencia que, antes del 1 de junio de 2009, diseñe una política que permita involucrar y comprometer efectivamente a las entidades territoriales en la superación del estado de cosas inconstitucional (...) Dicha política deberá ser formalizada mediante un acto administrativo." ${ }^{42} \mathrm{Al}$ respecto la sentencia T-1030-06: "Con el fin de verificar el cumplimiento de esta orden, la Secretaría de Educación Departamental de Sucre deberá remitir, dentro de la semana siguiente al vencimiento del plazo anteriormente señalado, copia del documento que contenga dicha política pública".
} 
administrativo o en una ley, aunque "puede concretarse también en normas de rango superior o inferior a los mencionados, pero generalmente se emplean estos dos actos jurídicos"43.

\section{El papel del legislador en las políticas públicas}

Pese a que se ha sugerido que "la ley no es una política pública" (Cuervo, 2007, p.77) y que en ella más bien se encuentran lineamientos de política pública, en algunas decisiones consultadas no parece tan clara la distancia del legislador respecto a su diseño o la exclusividad del gobierno para ello. Parecen identificarse dos situaciones, en la primera la Corte coincide con lo señalado por Cuervo (2007), es decir que la ley está primero que la política, es su marco y por eso no la sustituye, de modo que su formulación es tarea del ejecutivo:

De manera general, puede decirse que una ley, expedida por el Congreso de la República, preside el desarrollo de la política pública mediante la cual se busca la efectividad de un determinado derecho asistencial. Esta ley fija los parámetros generales de actuación del Estado y los criterios genéricos de distribución que sean necesarios de acuerdo con la naturaleza de cada derecho. (T-533-92)

La sentencia T-025-04 conserva la misma comprensión de la ley como marco general y de la política como su reglamentación, aunque en este caso parece radicar la función del diseño de la política en cabeza del legislador:

[...] no se trata en este caso de que a través de la acción de tutela se esté ordenando un gasto no presupuestado o que se esté modificando la programación presupuestal definida por el Legislador. Tampoco está definiendo nuevas prioridades, ni modificando la política diseñada por el Legislador y desarrollada por el Ejecutivo. (Cursiva propia)

En otra decisión la Corte parece sostener una relación complementaria entre la ley y la política pública al punto de considerar deseable que la ley se materialice en esta:

Bien puede ocurrir que un instrumento jurídico carezca de sustento en una política pública, y, quizás, eso es lo más frecuente. Ello, aunque indeseable, no torna el instrumento jurídico en inconstitucional, per se. Simplemente, lo hará más vulnerable a futuros juicios donde se analice su razonabilidad en la medida en que la relación entre los medios jurídicos y los fines de política pública será más remota, inasibley, probablemente, inadecuada (C-646-01).

\footnotetext{
${ }^{43}$ Hace parte de: Corte Constitucional, Sentencia C-646-01. M.P. Manuel José Cepeda.
} 
En esta primera situación, el camino adecuado que a juicio de la Corte debería seguir una ley, es ser desarrollada por el ejecutivo mediante una política pública a partir de los lineamientos legislativos. La política, por tanto, hace parte de la implementación de la ley ${ }^{44}$.

En la segunda situación hallada en otro grupo de decisiones, la Corte estima que en ciertos temas existe una especie de reserva de ley para formular la política pública. Este escenario aparece respecto a las políticas de seguridad y defensa y frente a la política criminal. Así, frente a la primera materia expresa: "corresponde a las mayorías representadas en el Congreso adoptar las políticas de seguridad y defensa que juzguen más adecuadas" (C-251-02). En similar sentido, la Corte aclara que la etapa de ‘diseño’ de la política criminal incluye una etapa legislativa. $\mathrm{Al}$ respecto sostiene:

La articulación jurídica de una política debe, racionalmente, ser antecedida de la definición de sus elementos constitutivos, de las metas, y de las prioridades. Sin embargo, ello no significa que la articulación legislativa de una política esté excluida de la etapa de diseño de la política. (C-646-01)

En la sentencia C-873-2003 la Corte confirma que la etapa legislativa hace parte del diseño de la política criminal y que también hacen parte de tal etapa los actos legislativos que el Congreso promulgue sobre la materia.

\section{Conclusiones}

La referencia a las políticas públicas en la jurisprudencia de la Corte Constitucional fue tímida durante los primeros años y se incrementó notablemente en los años 2008 y 2009, que corresponden a un mayor activismo de la Corte frente al derecho a la salud y al aumento en los autos de seguimiento de la sentencia T-025 de 2004. Adicionalmente, este periodo corresponde a un mayor protagonismo del magistrado Manuel José Cepeda Espinosa en el tema ${ }^{45}$.

En la Tabla 1 se presenta la distribución de las sentencias consultadas por año. Llama la atención que 63 de las decisiones que involucran políticas públicas son sentencias

\footnotetext{
${ }^{44}$ Al respecto la sentencia C-873-03 señala: "La "implementación" de una norma hace referencia al proceso por medio del cual la política que dicha norma articula jurídicamente es puesta en ejecución; se trata de una serie ordenada de pasos, tanto jurídicos como fácticos, predeterminados por la misma norma -o por aquellas que la desarrollen-, encaminados a lograr la materialización, en un determinado período de tiempo, de una política pública que la norma refleja."

${ }^{45}$ En efecto, trece de las 94 providencias consultadas son de ponencia de Manuel José Cepeda, le siguen Córdoba Triviño, Humberto Sierra Porto, Luis Ernesto Vargas y María Victoria Calle con ocho, luego Eduardo Cifuentes y Jorge Iván Palacio con siete. El resto de las decisiones se distribuye en pocos números entre varios magistrados.
} 
de tutela, fallos que en principio, solo involucran a las partes interesadas en el litigio particular pero que la Corte ha privilegiado como escenario de referencia al tema.

Tabla 1. Sentencias por año

\begin{tabular}{|l|c|c|}
\hline Año & Totales (T, SU, C, Auto) & Tutela \\
\hline 1992 & 1 & 1 \\
\hline 1995 & 1 & 1 \\
\hline 1997 & 1 & 0 \\
\hline 1998 & 4 & 2 \\
\hline 1999 & 3 & 3 \\
\hline 2001 & 1 & 0 \\
\hline 2002 & 4 & 2 \\
\hline 2003 & 3 & 2 \\
\hline 2004 & 4 & 3 \\
\hline 2005 & 2 & 2 \\
\hline 2005 & 1 & 0 \\
\hline 2006 & 9 & 9 \\
\hline 2007 & 7 & 4 \\
\hline 2008 & 13 & 12 \\
\hline 2009 & 12 & 7 \\
\hline 2010 & 9 & 3 \\
\hline 2011 & 8 & 4 \\
\hline 2012 & 7 & 6 \\
\hline 2013 & 4 & 2 \\
\hline TOTAL & $\mathbf{9 4}$ & \\
\hline & & $\mathbf{6 3}$ \\
\hline
\end{tabular}

Los hallazgos sobre la narrativa jurisprudencial alrededor de las políticas públicas permiten arribar a tres conclusiones fundamentales relacionadas con las hipótesis planteadas al inicio del texto.

La primera hipótesis, según la cual el principal escenario fáctico en el que se usa la categoría de políticas públicas en la jurisprudencia constitucional es el de la violación a la faceta prestacional y progresiva de los derechos constitucionales fue verificada. En efecto, de las 76 decisiones de tutela, sentencias de unificación y autos, todas aluden a esta faceta de los derechos, es decir, aquella que requiere acciones, recursos, planeación y plazos para su cumplimiento.

Lo anterior sugiere que la Corte logró relacionar algo que en principio no aparece relacionado en la Constitución de 1991: la carta de derechos y el modelo económico constitucional, lo que no indica que este diálogo germinal entre derechos y políticas públicas al interior de su jurisprudencia zanje la discusión entre eficiencia y derechos que aparece en 
el trasfondo del debate, teniendo en cuenta, además, la reciente incorporación del principio de sostenibilidad fiscal en la Constitución colombiana. Es un avance, en todo caso, considerar que la inexistencia o la deficiencia de las políticas, constituye un hecho violatorio de los derechos y que postergar su ejecución es un indicio de inconstitucionalidad ${ }^{46}$.

En la Tabla 2 se presenta la distribución de las sentencias T, SU y los Autos por derecho protegido.

\section{Tabla 2. Sentencias por derecho ( $T$, SU y Autos) $)^{47}$}

\begin{tabular}{|l|c|}
\hline Vivienda digna & 22 \\
\hline Salud & 17 \\
\hline Mínimo vital & 8 \\
\hline Igualdad & 4 \\
\hline Vida digna & 6 \\
\hline Autonomía indígena/Consulta previa & 3 \\
\hline Agua & 2 \\
\hline Trabajo & 2 \\
\hline Alimentación & 1 \\
\hline Derechos de los niños & 1 \\
\hline Libertad de expresión & 1 \\
\hline Seguridad Social & 1 \\
\hline TOTAL & $\mathbf{7 6}$ \\
\hline
\end{tabular}

La razón por la cual vivienda digna encabeza la lista de decisiones que se refieren de alguna manera al concepto de políticas públicas, es porque se trata del derecho principalmente protegido a la población en situación de desplazamiento, cuyo número de sentencias es el más elevado como se aprecia en la Tabla 3.

La segunda hipótesis era que el uso jurisprudencial de las políticas públicas obliga principalmente al gobierno y no al legislador, como competente para su diseño. En la mayor parte de las fuentes consultadas la Corte atribuye la obligación de diseñar políticas públicas al ejecutivo aunque no descarta la intervención del legislador con el fin de 'enmarcar' la política. Por su parte, las órdenes en materia de políticas públicas que señala la Corte en la parte resolutiva de sus providencias, se dirigen al gobierno, ya sea en el nivel central o a nivel territorial.

\footnotetext{
${ }^{46}$ Así lo advierte la Corte en sentencia T-411-09: "es inconstitucional que las autoridades posterguen de manera indefinida -o hasta que el Estado cuente con los recursos suficientes y la capacidad administrativa adecuada- el cumplimiento y ejecución de políticas públicas que estén relacionadas con el avance gradual y progresivo de un derecho".

${ }^{47}$ Esta clasificación se hizo según el derecho que la Corte protege y no el que invoca el demandante en la acción de tutela. En algunos casos la Corte protege más de un derecho, sin embargo se eligió aquél sobre el cual gira la argumentación principal de la sentencia.
} 
La tercera hipótesis era que las políticas públicas son asimiladas en la jurisprudencia de la Corte a conceptos cercanos como políticas de Estado, planes y programas. Tal como se advirtió, la jurisprudencia estudiada asimila las políticas públicas a conceptos cercanos y se refiere a estos términos de manera complementaria con el fin de reforzar su propósito.

Otra conclusión a la que es posible arribar con la investigación tiene que ver con los destinatarios de las políticas públicas. Es claro que la Corte privilegia las políticas como herramienta para la garantía de los derechos de personas en condición de debilidad manifiesta. Veamos la distribución de la población protegida en las fuentes consultadas:

\section{Tabla 3. Sentencias por población (T, SU y Autos)}

\begin{tabular}{|l|c|}
\hline Población desplazada & 19 \\
\hline Población discapacitada & 11 \\
\hline No población vulnerable & 11 \\
\hline Niños y niñas & 9 \\
\hline Vendedores ambulantes & 8 \\
\hline Personas privadas de la libertad & 5 \\
\hline Ancianos & 2 \\
\hline Indígenas/Afrodescendientes & 3 \\
\hline Recicladores & 2 \\
\hline Indigentes & 1 \\
\hline Población LGBTI & 1 \\
\hline Personas en zonas de alto riesgo & 1 \\
\hline Personas con VIH- Sida & 1 \\
\hline Mujeres & 1 \\
\hline Bicitaxistas & 1 \\
\hline Total & $\mathbf{7 6}$ \\
\hline
\end{tabular}

En síntesis, la narrativa jurisprudencial de las políticas públicas ha tenido la intención de presionar la acción gubernamental frente a los problemas públicos. Tal presión ha ocurrido, entre otras vías, por medio del "efecto simbólico" (Rodríguez Garavito y Rodríguez Franco, 2010) de las decisiones judiciales que consiste en propiciar el cambio en las "ideas, percepciones e imaginarios sociales sobre el tema objeto de litigio" (Rodríguez Garavito y Rodríguez Franco, 2010, p. 97) a través de un lenguaje cargado de términos que invitan a la acción como el que utiliza la Corte en su narrativa.

Sin embargo, la narrativa jurisprudencial sobre los escenarios y la formalización de las políticas públicas no es unívoca ni coherente, frente a lo cual la pregunta obligada es ¿sería deseable que tal narrativa fuera homogénea y que la Corte precisara su doctrina en la materia? En principio se podría argumentar que la uniformidad de las decisiones no es exigible a la Corte en tanto cada caso decidido tiene sus contornos y, además, la variedad de tendencias en su narrativa hace parte de la dinámica del derecho. Desde esta mirada, cerrar la interpretación de la Corte sobre el concepto de política pública 
no solo es indeseable sino además imposible, porque por un lado, muchas decisiones de la Corte son tomadas en salas de revisión de tres magistrados y no en sala plena y, por otro lado, es posible apartarse del precedente judicial argumentando suficientes razones para ello, por lo cual las decisiones de la Corte son circunstanciales y revocables.

Sin embargo, algunas precisiones jurisprudenciales serían convenientes por las siguientes razones:

1. Por un lado, la alusión a las políticas públicas ha dependido más de liderazgos individuales de algunos magistrados - principales y auxiliares- que se han puesto en la tarea de ingresar teóricamente a este campo disciplinar, por lo cual una vez se pierden estos liderazgos por su salida de la Corte, el concepto cae nuevamente en la discusión sobre si la Corte debe o no intervenir en políticas públicas.

2. Por otro lado, en las audiencias públicas ante el Senado para la elección de magistrados a la Corte Constitucional, una de las preguntas clave para los candidatos ha sido si consideran que la Corte debe intervenir en políticas públicas o no debe hacerlo. Los candidatos han sido tímidos en sus respuestas porque alrededor del tema se mantiene el temor del gobierno de los jueces (López, 2011) ${ }^{48}$. Parte de esta prevención hacia el poder judicial cuando se involucra en el campo de las políticas, podría disminuir si se aclara, como lo ha hecho la Corte en algunas de las sentencias aquí analizadas, los escenarios bajo los cuales opera tal intervención -la faceta progresiva de los derechos y el estado de cosas inconstitucional-y su competencia en la materia que, en ninguno de los casos estudiados, llegó a reemplazar la voluntad del policy maker.

3. Finalmente, una razón más que amerita alguna claridad conceptual sobre el uso de las políticas públicas en la Corte, es la necesidad de enviar un mensaje más preciso a las autoridades destinatarias de las órdenes estructurales ${ }^{49}$, a los organismos de control vinculados para hacer seguimientos a este tipo de sentencias ${ }^{50}$ y a los grupos interesados en el litigio estructural ante esa Corporación.

\footnotetext{
${ }^{48}$ En la elección de 2008 se le preguntó a los candidatos: "¿Cuál sería en su criterio el alcance de las competencias de la jurisdicción constitucional en el diseño y la evaluación de políticas públicas en el marco de las declaratorias de Estado de Cosas Inconstitucional, teniendo en cuenta los efectos presupuestales e institucionales que ello genera?". Otra pregunta similar que se les formuló fue: "¿Cree usted que la Corte Constitucional es competente para intervenir en el diseño, implementación y ejecución de políticas públicas con el fin de que estas garanticen el goce efectivo de los derechos fundamentales de sus beneficiarios?" Disponible en: http://www.eleccionvisible.com/index.php?option=com_content\&view=frontpage\&Itemid=1 ${ }^{49}$ En decisiones recientes la Corte ha optado por indicar los parámetros para el diseño de las políticas. La parte resolutiva de la sentencia T- 442-13 lo ilustra cuando ordena "Exhortar a la Alcaldía Mayor de Bogotá para que [...] proceda a diseñar la política a nivel local sobre el bicitaxismo, teniendo en cuenta los parámetros señalados en esta sentencia".

50 Un ejemplo de ello es la sentencia T-1089-12 que indica: "se remitirá copia de la presente sentencia con destino a la Defensoría del Pueblo para que, en ejercicio de sus funciones, vigile la
} 
Volviendo a la idea de Majone (2005) según la cual la política pública está hecha de palabras, esta investigación intentó hilar los relatos de la Corte Constitucional colombiana sobre este concepto, a partir de las circunstancias o el contexto en que se refiere a las políticas y de su formalización legal. Aunque no es seguro el rumbo que tomarán los nuevos magistrados que van llegando a esa corporación frente al tema, quedo claro que en más de veinte años de operación, la Corte Constitucional ha encontrado en la categoría de políticas públicas un interesante concepto para obligar a las autoridades a emprender acciones que garanticen la faceta programática de los derechos constitucionales. Con esto, en lugar de sustituir la voluntad democrática, la Corte llama a rendir cuentas a los órganos competentes. Tal como indica Langford (2013) "esto apunta a cómo los tribunales se están configurando como mecanismos de control de los Estados, y no como mecanismos para elaborar políticas públicas," (p.101) de este modo se le sale al paso a quienes critican el activismo desbordado de la Corte en materia política (politics) y de políticas (policies). Los escenarios y la formalización del concepto de políticas públicas de la Corte, asuntos aquí explorados, indican que su narrativa jurisprudencial está lejos de querer sustituir la voluntad democrática o de buscar un gobierno de los jueces.

\section{Referencias}

Abramovich, V. y Pautassi, L. (Comps.). (2009). La revisión judicial de las políticas sociales. Buenos Aires: Del puerto.

Cano Blandón, L. F. (2012). Corte Constitucional y políticas públicas. ¿́fortalecimiento o riesgo para la Democracia? En M. Montoya y Restrepo, N. (Eds.). Reflexiones constitucionales. A propósito de dos décadas de la Constitución en Colombia (pp. 267-295). Medellín: Ed. Universidad Eafit.

Cuervo, J. (2007). Las políticas públicas entre los modelos teóricos y la práctica gubernamental. En Ensayos sobre políticas públicas (pp. 65-96). Bogotá: Universidad Externado de Colombia.

Dahl, R. (1957). Decision-making in a Democracy. The Supreme Court as a national policy maker. Journal of Public Law, 6, 279-295.

Fischer, F. (1996). Policy Discourse and the politics of Washington think tanks. En F. Fischer y J. Forester (Eds.). The argumentative turn in Policy Analysis and Planning (pp. 21-40). Duke: Duke University Press.

política pública que se viene adelantando en el municipio de Tabio para garantizar la efectividad del derecho fundamental al agua de los demandantes". 
Fischer, F. (2007). Deliberative policy analysis as practical reason: Integrating empirical and normative arguments. En F. Fischer y G. Miller (Eds.). Handbook of public policy. Theory, politics and methods (pp. 223-236). Boca Raton: CRC Press.

Gottweis, H. (2007). Rhetoric in policy making: Between logos, ethos, and pathos. En F. Fischer y G. Miller (Eds.). Handbook of public policy. Theory, politics and methods (pp. 237-250). Boca Raton: CRC Press.

Langford, M. (2013). La exigibilidad judicial de los derechos sociales: de la práctica a la teoría. En M. Langford (Ed.). Teoría y jurisprudencia de los derechos sociales (pp. 45-104). Bogotá: Universidad de los Andes.

López Daza, G. (2011). El juez constitucional colombiano como legislador positivo: ¿un gobierno de los jueces? Revista Mexicana de Derecho Constitucional, 24, 169-193.

Majone, G. (2005). Evidencia, argumentación y persuasión en la formulación de políticas públicas. México: Fondo de Cultura Económica.

Rodríguez Garavito, C., Arcidiácono, P. y Espejo, N. (Coords.). (2010). Derechos Sociales: justicia, política y economía en América Latina. Bogotá: Siglo del Hombre.

Rodríguez Garavito, C. y Rodríguez Franco, D. (2010). Un giro en los estudios sobre derechos sociales: el impacto de los fallos judiciales y el caso del desplazamiento forzado en Colombia. En: P. Arcidiácono, N. Espejo, y C. Rodríguez (Coords.). Derechos Sociales: justicia, política y economía en América Latina (pp. 83-154). Bogotá: Siglo del Hombre.

Roe, E. (1992). Applied narrative analysis: the tangency of literary criticism, social science and policy analysis. New Literary History, 23(3), 555-581.

Roe, E. (1994). Narrative policy analysis: theory and practice. Duke: Duke University Press.

Roth, A. N. (2006). Políticas públicas. Bogotá: Ed. Aurora.

Roth, A. N. (2007). Enfoques y teorías para el análisis de las políticas públicas, cambios de la acción pública y transformaciones del Estado. En Ensayos sobre políticas públicas (pp. 27-64). Bogotá: Universidad Externado de Colombia.

Roth, A. N. (2010). Las políticas públicas y sus principales enfoques analíticos. En A. Roth (Ed.). Enfoques para el análisis de políticas públicas (pp. 17-64). Bogotá: Universidad Nacional de Colombia.

Sierra Cadena, G. (2009). El juez constitucional: un actor regulador de las políticas públicas. El caso de la descentralización en Colombia. Bogotá: Universidad del Rosario.

Subirats, J. y Knoepfel, P. (2008). Análisis y gestión de políticas públicas. Barcelona: Ariel. 
Van Eeten, M. (2007). Narrative Policy Analysis. En F. Fischer y G. Miller (Eds.). Handbook of public policy. Theory, politics and methods (pp. 251-269). Boca Raton: CRC Press. 